\title{
BIOQ-BYWORD
}

\section{To Explore the Effect of Evidence-Based Nursing Care in the Elderly Patients with Pressure Sore Care}

\author{
Shen Li
}

Yangzhou Community Health Service Center, Yangzhou City, Jiangsu Province, Yangzhou 225000

\section{ARTICLE INFO}

\section{Article history:}

Published online: $30^{\text {th }}$ Sept, 2017

\section{Key words:}

Routine care

Evidence-based care

Elderly patients with chronic diseases and advanced cancer

\begin{abstract}
Objective: To summarize the experiences of the elderly patients with chronic diseases and the patients with advanced cancer. Methods: From January 2014 to March 2015, 114 patients with advanced disease and advanced cancer were enrolled in our hospital. The patients were divided into observation group and control group. The control group was treated with routine nursing and observation group on evidence-based care. To observe and compare the two groups of patients with pressure ulcers and the severity of pressure sores. Results: The incidence of pressure sore was $7.02 \%$ in the observation group and $33.33 \%$ in the control group. The incidence of pressure sore was significantly lower than that of the control group, the difference was statistically significant $(\mathrm{P}<0.05)$. Conclusion: Evidence-based care in elderly patients with chronic diseases and advanced cancer patients can reduce the occurrence of pressure sores and reduce the severity of pressure sores, which has a high application value.
\end{abstract}

\section{Introduction}

For the elderly patients with chronic diseases and advanced cancer patients, pressure sores is one of the most common complications, which seriously affects the quality of life of patients. In order to verify the effect of evidence-based nursing care in the treatment of pressure sores, this article specializes in 114 elderly patients as the research object, 57 cases of routine care, 57 cases of evidence-based care, after the nursing intervention to judge the effectiveness of their intervention. Now introduced as follows.

\section{Materials and methods}

\subsection{General information}

In this study, 114 elderly patients with chronic diseases and advanced cancer were enrolled in this study. The time span was from January 2014 to March 2015, with 57 cases divided into observation group and control group.
Observation group: 25 males and 32 females; aged 60-94 years, mean $75.5 \pm 5.6$ years old. Control group: 26 males and 31 females; aged 59-89 years, mean $76.5 \pm 4.2$ years old. Inclusion criteria ${ }^{[1]}$ : Braden scores no more than 13 points; admission without pressure sores. Exclusion criteria: less than 3 days of admission. There was no significant difference between the two groups $(\mathrm{P}>0.05)$.

\subsection{Methods}

\subsubsection{Control group routine care}

1) Keep the bed dry and clean.

2) Strengthen nutritional support.

3) Patients in the air bed in the supine or lateral position, change 1 position in every $2 \mathrm{~h}$ to ensure effective decompression; In necessary circumstances, every $2 \mathrm{~h}$ raised the buttocks and compression site 1 .

4) As far as possible to reduce skin friction, rub every day to ensure that skin hygiene ${ }^{[2]}$.

\subsubsection{Observation group evidence-based care}


The collection and statistics of the elderly patients with a pressure sore in the implementation of the problems encountered. The main issues reported to the higher authorities to clear the existing problems and enhance the degree of attention; search authority to report the literature and support the evidence to report the issue; organize the relevant physicians and experts to review the dissemination of scientific evidence and efficiency ${ }^{[3]}$; In the review of effective, widely representative of the evidence under the guidance of the development of nursing programs and give full consideration to patient care needs and practical nursing experience to ensure that the care plan is practical and effective ${ }^{[5]}$; Strict implementation of the nursing plan. If necessary, it can be adjusted appropriately. Specific methods are as follows:

1) Admission is the assessment, followed by the patient's skin to conduct a dynamic assessment of the patient's skin dynamic observation. Record the Braden score and in the event of disease changes immediately re-assessment.

2) Every two h turn over once. The disease allows the case to keep the bed below 30 degrees. The body placed in a special position to support the body does not move or slide to avoid compression at the bone.

3) Use of infantile shower gel and other ingredients containing cream and warm water on the patients to rub the bath, to avoid irritating lotion and cold water; For the skin chapped, scaling of patients, can first use baby oil smear, 30min (Borneol, Catechu, Cork, Scutellaria, 75\% ethanol in proportion to the proportion of configuration), if necessary, can increase the number of times and the amount of the use of the skin, Edema at the skin forbidden massage; The water colloid dressings attached to high-risk groups may be pressure parts ${ }^{[4]}$.

4) Nutritional support: The establishment of intravenous access to ensure that sugar, vitamins, protein, fat and a variety of trace elements can be a timely supply of patients to enhance the body defense system. If severe malnutrition, it may be appropriate to add fresh plasma, compound amino acids, albumin into diet; Mouth to eat patients with enteral nutrition suspension.
5) For fecal incontinence or frequent diarrhea patients, the perineum is the focus of care. It should be promptly treated with warm water. Female patients have to clean the labia and wrinkles; Use OB cotton saddle, skin protective film or an anorectal pouch to protect the perianal skin and the use of $\mathrm{OB}$ sliver to do the timely replacement; The use of anorectal pouch to do timely discharge bag feces and ensure that the perianal skin paste close $^{[5]}$.

6) Psychological care: As far as possible so that patients and their families involved in the pressure of the possibility and severity of the assessment process. To introduce the basic knowledge of pressure sores and inform the basic care methods and common trigger factors to enhance their self-protection and the ability to self-care; To patients with similar cases to cure have to inform the importance of their care; With its more communication, the timely guidance of bad mood.

\subsection{Observe indicators}

The incidence of pressure sores, the severity of pressure sores.

\subsection{Statistical methods}

SPSS 20.0 statistical software was used to analyze the specific data obtained by the statistics, all the count data with $\mathrm{n} / \%$ said, compared with the test, if $\mathrm{P}<0.05$, then the results were statistically significant.

\section{Results}

\subsection{Comparison of the incidence of two groups of pressure sores}

The incidence of pressure ulcer in the observation group was lower than that in the control group, the difference was statistically significant $(\mathrm{P}<0.05)$. See Table 1 for details.

Table 1 Comparison of the incidence of two groups of pressure sores

\begin{tabular}{cccc}
\hline Group & Number of case & Occurrence of pressure sore/case & Rate of occurrence of pressure sore/\% \\
\hline Control group & 57 & 19 & 33.33 \\
Observation group & 57 & 4 & 7.02 \\
\hline
\end{tabular}

\subsection{Comparison of the severity of the two groups of pressure sores}

Observation group were I pressure sores; control group
$78.95 \%$ is I stage pressure sores, $21.05 \%$ is phase II pressure sores. The difference was statistically significant $(\mathrm{P}<0.05)$. See Table 2 for details.

Table 2 Comparison of the severity of the two groups of pressure sores

\begin{tabular}{cccc}
\hline Group & Occurrence of pressure sore/case & Phase I pressure sores $(\mathrm{n}, \%)$ & Phase II pressure sores $(\mathrm{n}, \%)$ \\
\hline $\begin{array}{c}\text { Control group } \\
\begin{array}{c}\text { Observation } \\
\text { group }\end{array}\end{array}$ & 19 & $15(78.95)$ & $4(21.05)$ \\
\hline
\end{tabular}




\section{Discussion}

Elderly patients with chronic diseases and advanced cancer patients need to stay in bed for a long time. In addition to suffering from physical damage, the patient's psychological will suffer a great torture, which is very prone to do not cooperate with the phenomenon of medical work. Evidence-based care not only concerned about the patient's physical needs, it can balance their mental state and reduce the negative emotions. The key to the implementation of evidence-based care is:

1) Master the basic situation of patients, such as education, character, occupation, etc., for patients to develop an appropriate communication plan. Pay attention to communication with patients to keep abreast of their psychological state. Negative emotions need immediately to ease, so that patients with the disease have a correct understanding of the patient as soon as possible into the role to encourage patients to establish the confidence to overcome the disease. Enhance their psychological experience and compliance to facilitate the development of our health care work.

2) The patient's skin condition dynamic observation and the disease changes in time to assess and record the score.

3) Focus on patient skin care to ensure that the skin clean and reduce unnecessary friction ${ }^{[6]}$.

4) Should stand up and master the correct way to turn over to avoid a long time on a part of the pressure.

5) Pay close attention to pressure sores and take certain measures to deal with to avoid the further development of complications. In this care mode, caregivers care behavior strictly based on scientific laws and principles, rather than just a simple personal intuition and experience. Relevant studies have pointed out ${ }^{[7]}$,evidence-based care can reduce the risk of pressure ulcers in critically ill patients. In this study, the incidence of pressure ulcer was $7.02 \%$ in the observation group, which was significantly lower than that in the control group (33.33\%), and the difference was statistically significant $(\mathrm{P}<0.05)$, suggesting that evidence-based nursing can reduce the incidence of pressure sores, which consistent with the above point of view. In addition, evidence-based nursing is also conducive to the improvement of the overall quality of nursing staff, the mastery of nursing knowledge more comprehensive, professional, scientific, learning awareness and service awareness will be significantly improved. It is conducive to the improvement of nursing satisfaction ${ }^{[8]}$. In general, evidence-based care in elderly patients with chronic diseases and advanced cancer patients can

reduce the incidence of pressure sores and reduce the severity of pressure sores, which has a high application value.

\section{References}

[1] Duan Yushun. Emergency Care in Patients with Severe Pressure Ulcer Prevention and Treatment of Nursing Experience. Medical Information, 2013, 26(15): 340-341.

[2] Han Limei, Lu Xiaoxia. One Case of Right Rear Hip Pressure Ulcer Associated with Left Pneumonia in Patients with Nursing Experience. Chinese poisoning treatment of the Capital Forum - and the sixth national poisoning and critically ill medical conference Proceedings. 2014, 350-351.

[3] Qian Xi Ming. Intensive Care of Patients with Pressure Ulcers Nursing Experience. Medical aesthetic beauty (mid-term), 2014, 8: 390-391.

[4] Wang Yang. Skin Care in Critically Ill Patients. Proceedings of the Third World Disaster Care Conference. 2014, 193-193.

[5] Xiao Sha, Liang Fu Xiang. Pressure Ulcer Care Group in Elderly Critically Ill Patients with Pressure Ulcer Prevention and Nursing Role in the Study. Guangzhou Medical College, 2013, 41 (5): 107-108. 